\title{
A critical appraisal of once-daily topical luliconazole for the treatment of superficial fungal infections
}

This article was published in the following Dove Press journal:

Infection and Drug Resistance

18 January 2016

Number of times this article has been viewed

\author{
Aditya K Gupta ${ }^{1,2}$ \\ Deanne Daigle² \\ 'Department of Medicine, University \\ of Toronto School of Medicine, \\ Toronto, ${ }^{2}$ Dermatology, Mediprobe \\ Research Inc., London, ON, Canada
}

\begin{abstract}
Luliconazole is a novel imidazole derivative, which has demonstrated in vitro efficacy against dermatophytes and Candida. The results from Phase III trials show that luliconazole 1\% cream applied once daily for 2 weeks successfully resolved the clinical signs and symptoms as well as eradicated the pathologic fungi, which cause tinea pedis. A 1-week treatment with luliconazole $1 \%$ cream also produced favorable clinical and mycological results in clinical trials for tinea corporis and tinea cruris. Across trials, adverse events consisted mainly of localized reactions following application. The development of a new antifungal agent is timely due to mounting resistance among existing treatments. Because luliconazole requires a short duration of treatment, it may assist in reducing disease recurrence as a result of patient nonadherence. Keywords: antifungal, dermatophyte, athlete's foot, tinea cruris, tinea corporis, imidazole
\end{abstract}

\section{Introduction}

Tinea are superficial fungal infections typically caused by dermatophytes. ${ }^{1}$ Common pathogens include Trichophyton rubrum, Trichophyton mentagrophytes, and Epidermophyton floccosum. ${ }^{1}$ Superficial fungal infections are widespread, with an estimated worldwide prevalence of $20 \%-25 \%$, and include tinea pedis (athlete's foot), tinea cruris (jock itch), and tinea corporis (ringworm), among others. ${ }^{2}$ Tinea of the skin presents clinically as well-demarcated, scaling, and inflamed lesions, which are often accompanied by an itching or burning sensation. ${ }^{3}$ In the case of tinea pedis, maceration and vesiculation may also be present. Potassium hydroxide (KOH) microscopy, fungal culture, or examination under a Wood's lamp can confirm the diagnosis of cutaneous dermatophyte infections. Some cases can be chronic and may lead to more extensive dermatophytosis if left untreated. ${ }^{4,5}$ Most mild-to-moderate tinea infections can be treated with topical antifungals, while oral agents are usually reserved for severe or recalcitrant cases. ${ }^{1}$

Topical allylamine (terbinafine and butenafine) and imidazole (clotrimazole and bifonazole) antifungals are popular treatment choices for infections of the feet, body, and groin. Both allylamine and imidazole antifungals work by inhibiting the synthesis of ergosterol, an essential component of the fungal cell membrane. ${ }^{6}$ Allylamines block the conversion of squalene into squalene-2,3-epoxide, a precursor of ergosterol formation, while imidazoles selectively inhibit fungal cytochrome P450 (CYP450) $14 \alpha$-demethylase enzyme, which disrupts the conversion of lanosterol to ergosterol. ${ }^{7}$ Imidazoles also alter the synthesis of triglycerides and phospholipids, which leads to an accumulation of toxic levels of hydrogen peroxide within fungal cells. ${ }^{7}$ This buildup of toxic concentrations of hydrogen peroxide may contribute to the deterioration of
Correspondence: Aditya K Gupta Mediprobe Research Inc., 645 Windermere Road London, ON N5X 2PI, Canada

Tel + I 5198579715

Fax + I 5196574233

Email agupta@execulink.com
Dovepress

(c) (i) (9) 2016 Gupta and Daigle. This work is published and licensed by Dove Medical Press Limited. The full terms of this license are available at https://www.dovepress.com/ terms.php and incorporate the Creative Commons Attribution - Non Commercial (unported, v3.0) License (http://creativecommons.org/licenses/by-nc/3.0). By accessing the work you permission for commercial use of this work, please see paragraphs 4.2 and 5 of our Terms (https://www.dovepress.com/terms.php). 
subcellular organelles and to cell death. ${ }^{7}$ Imidazoles have been shown to inhibit the transformation of blastospores of Candida albicans into the invasive mycelial form; thus, rendering them more favorable than allylamines for the treatment of superficial infections caused by Candida., However, current imidazole antifungals have some limitations: conventional use has produced fungal resistance and many agents require treatment courses lasting several weeks, which can contribute to patient nonadherence, and ultimately, disease recurrence. ${ }^{9}$

Luliconazole $\{(-)-E$-[(4R)-4-(2,4-dichlorophenyl)-1,3dithiolan-2-ylidene] (1H-imidazol- 1-yl) acetonitrile $\}$ is a novel imidazole antifungal. ${ }^{10}$ Luliconazole is uniquely characterized by its R-enantiomer side chain in addition to one chiral center. ${ }^{11}$ The addition of an imidazole moiety into the ketene dithioacetate structure of the compound augments its ability to target filamentous fungi, such as dermatophytes, without compromising its broad-spectrum antifungal activity. ${ }^{12}$ Luliconazole has been shown to have antifungal activity against dermatophytes and Candida in vitro, and has been clinically assessed for the treatment of tinea pedis, cruris, and corporis.

\section{Luliconazole I\% cream}

The pharmacokinetics of luliconazole $1 \%$ cream were investigated in 12 participants with moderate to severe tinea pedis and eight participants with moderate to severe tinea cruris. ${ }^{11}$ The participants applied luliconazole 1\% cream once daily for 15 days. Luliconazole plasma concentrations were measurable in all participants on day 15 and varied little within the 24-hour interval. The mean \pm standard deviation of the maximum concentration $\left(\mathrm{C}_{\max }\right)$ was $0.40 \pm 0.76 \mathrm{ng} / \mathrm{mL}$ and $4.91 \pm 2.51 \mathrm{ng} / \mathrm{mL}$ after the first dose, and $0.93 \pm 1.23 \mathrm{ng} / \mathrm{mL}$ and $7.36 \pm 2.66 \mathrm{ng} / \mathrm{mL}$ after the final dose in the participants with tinea pedis and tinea cruris, respectively. The mean time to reach $\mathrm{C}_{\max }\left(\mathrm{T}_{\max }\right)$ was $16.9 \pm 9.39$ hours after the first dose and 5.8 \pm 7.61 hours after the final dose for the participants with tinea pedis, and was $21.0 \pm 5.55$ hours after the first dose and $6.5 \pm 8.25$ hours after the final dose in the participants with tinea cruris. In participants with tinea pedis, exposure to luliconazole as expressed by area under the concentration time curve $\left(\mathrm{AUC}_{0-24}\right)$ was $6.88 \pm 14.50 \mathrm{ng} * \mathrm{hr} / \mathrm{mL}$ after the first dose and $18.74 \pm 27.05 \mathrm{ng} * \mathrm{hr} / \mathrm{mL}$ after the final dose. Exposure to luliconazole, as expressed by $\mathrm{AUC}_{0-24}$ was $85.1 \pm 43.69 \mathrm{ng} * \mathrm{hr} /$ $\mathrm{mL}$ after the first dose and $121.74 \pm 53.36 \mathrm{ng} * \mathrm{hr} / \mathrm{mL}$ after the final dose in the participants with tinea cruris.

The antifungal activity of luliconazole has been compared to that of other commercially available agents in vitro. ${ }^{10,12,13}$
The minimum inhibitory concentration for luliconazole was 2-4 times lower than lanoconazole and almost equal to or marginally lower than terbinafine for T. rubrum and $T$. mentagrophytes strains (Table 1). Luliconazole was shown to have the highest antifungal activity against Trichophyton spp. of currently available topical antifungal drugs and was also highly effective against Candida. ${ }^{10,12,13}$ Luliconazole $1 \%$ was also found to successfully eradicate experimentallyinduced T. mentagrophytes infections in half the time or less required for $1 \%$ terbinafine cream and bifonazole $1 \%$ cream. ${ }^{14}$ Promising findings from preliminary studies lead to the clinical investigation of luliconazole's efficacy in treating tinea pedis, tinea cruris, and tinea corporis infections.

\section{Tinea pedis}

A randomized, double-blind, multicenter, Phase II trial examined the efficacy and safety of several doses of luliconazole cream for plantar and interdigital tinea pedis. ${ }^{15}$ A total of 241 participants with interdigital or plantar type tinea pedis were enrolled into one of three groups: luliconazole $1 \%$ (group A), luliconazole $0.5 \%$ (group B), or luliconazole $0.1 \%$ (group C). The participants were instructed to apply luliconazole once daily for 14 days. Study endpoints included improvement in cutaneous symptom scores from baseline, clinical efficacy, defined as the proportion of the participants whose symptoms were deemed to be "markedly" or "moderately" improved, the proportion of the participants with negative $\mathrm{KOH}$, and the rate of global improvement as judged by the study investigator. Clinical assessments and skin sampling were performed at days $0,14,28$, and 42 of the study. Adverse event (AE) documentation, tolerability, hematology, blood biochemistry, and urine tests were also performed at all visits.

A total of 213 participants were evaluated for efficacy. Improvement of clinical symptoms, as expressed by the rate of participants who had markedly or moderately improved, stabilized for all the participants by week 4 . Global improvement rates for groups $\mathrm{A}, \mathrm{B}$, and $\mathrm{C}$ were $87.8 \%, 94 \%$, and $88.8 \%$ at 6 weeks, respectively (Table 2). Separate analysis of the negative $\mathrm{KOH}$ rates for interdigital and plantar tinea pedis revealed a trend in the concentration-dependent relationship in the negative conversion rate of fungi $(P=0.038)$, with the $1 \%$ concentration demonstrating the highest negative conversion rates. Overall, five AEs were reported and consisted of eczema and contact dermatitis in one patient each of group A, one case of pruritus in group B, and dyshidrotic eczema, erythema, and pain in one participant each in group C. Based on the results from this dose-finding study, the $1 \%$ formulation of luliconazole cream was determined 
Table 2 Clinical efficacy* rates from a Phase II dose-finding study of luliconazole cream for tinea pedis

\begin{tabular}{llll}
\hline & $\begin{array}{l}\text { Luliconazole I\% } \\
\mathbf{N}=\mathbf{7 4}\end{array}$ & $\begin{array}{l}\text { Luliconazole } \\
\mathbf{N}=\mathbf{0 . 5} \%\end{array}$ & $\begin{array}{l}\text { Luliconazole } \mathbf{0 . 1 \%} \\
\mathbf{N}=\mathbf{7 2}\end{array}$ \\
\hline Week 2 & $52.7 \%$ & $44.8 \%$ & $45.1 \%$ \\
Week 4 & $79.7 \%$ & $74.6 \%$ & $72.2 \%$ \\
Week 6 & $87.8 \%$ & $94.0 \%$ & $88.8 \%$ \\
\hline
\end{tabular}

Note: *Defined as the proportion of participants whose symptoms were deemed to be "markedly" or "moderately" improved based on the change in investigator global assessment scores from baseline.

to be the most effective and safe concentration for further investigation in Phase III trials.

A total of 147 patients with $\mathrm{KOH}$ and culture-confirmed interdigital tinea pedis were enrolled in a randomized, double-blind, vehicle-controlled, Phase III trial of luliconazole $1 \%$ cream. ${ }^{16}$ The participants were randomized in a 2:2:1:1 ratio to 2- or 4-week treatment with luliconazole $1 \%$ cream or vehicle once daily. The primary endpoint was complete clearance, defined as both clinical cure (score of 0 on a 4-point physician global assessment [PGA] scale) and mycological cure (negative $\mathrm{KOH}$ and fungal culture) within 2 weeks following completion of treatment. Secondary efficacy endpoints included effective treatment (defined as the rate of participants who had a PGA of 0 or 1 , and negative $\mathrm{KOH}$ and fungal culture), clinical cure, mycological cure, and complete clearance, all at the end of treatment, and at 2 and 4-week posttreatment. The AEs were also documented throughout the course of the study. Complete clearance and mycological cure rates were measured 2- and 4-weeks posttreatment. Efficacy rates in both luliconazole 1\% groups were higher than vehicle when measured at 2- and 4-week posttreatment; however, no $P$-values were reported. There were no treatment-related AEs.

Another randomized, double-blind, vehicle-controlled Phase III study of luliconazole $1 \%$ cream was performed on 209 participants with interdigital tinea pedis. ${ }^{17}$ The participants were randomized in a $1: 1$ ratio to receive either luliconazole $1 \%$ cream or vehicle once daily for 2 weeks. Clinical signs and symptoms, as well as mycology ( $\mathrm{KOH}$ and culture) were conducted at days 28 and 42 of the study. The PGA was performed on days 14, 28, and 42 by an investigator using a 4-point scale ( 0 [none] to 3 [severe]). The primary efficacy endpoint was complete clearance, defined as both a clinical (total absence of signs and symptoms) and mycological cure (negative $\mathrm{KOH}$ and culture) at day 42. Secondary efficacy endpoints included the proportion of patients achieving clinical cure and mycological cure at day 42 , and complete clearance at day 28 
(2-weeks posttreatment). The AEs were also monitored and documented throughout the trial.

Luliconazole $1 \%$ cream was significantly more effective than vehicle in producing complete clearance at 2 - and 4-week posttreatment $(P=0.001$ and $P<0.001$ for 2 - and 4-week assessments, respectively). The participants treated with luliconazole $1 \%$ cream also had significantly higher mycological cure rates compared to vehicle when assessed at 2 weeks $(P<0.001)$; however, mycological cure rates at 4-week posttreatment follow-up were not reported. The most common treatment-related AEs were application-site reactions experienced by less than $1 \%$ of participants.

The efficacy of luliconazole $1 \%$ cream in treating interdigital and plantar-type tinea pedis was evaluated against an active comparator in a multicenter, randomized, singleblind, parallel group study. ${ }^{18} \mathrm{~A}$ total of 511 participants were assigned to luliconazole $1 \%$ cream once daily for 2 weeks and 2 -week vehicle, or bifonazole $1 \%$ cream for 4 weeks by block randomization method according to the type of tinea pedis. Skin sampling for $\mathrm{KOH}$ wet mount, as well as clinical ratings of signs and symptoms were performed at each visit. Study endpoints were evaluated at 4-week posttreatment and included overall cure (clinical and negative $\mathrm{KOH}$ ), clinical efficacy (defined as the rate of participants who exhibited marked or moderate symptom improvement from baseline), and mycological efficacy (negative $\mathrm{KOH}$ ).

Overall cure rates were similar between luliconazole 1\% and bifonazole $1 \%$ ( $74.1 \%$ vs $73.4 \%, P=0.918)$. Clinical and mycological cure rates also were comparable between treatment groups (clinical cure: $91.5 \%$ vs $91.7 \%, P=1$; mycological cure: $76.1 \%$ vs $75.9 \% ; P=1$, for luliconazole $1 \%$ cream vs bifonazole $1 \%$ cream, respectively). Treatmentrelated AEs were experienced by 5/253 (2.0\%) participants in the luliconazole $1 \%$ group and $6 / 247$ (2.4\%) participants in the bifonazole $1 \%$ group. The AEs in both groups were localized reactions and consisted of erythema, itching, pain, and redness in the luliconazole group and itching, erythema, skin inflammation, irritation, and redness in the bifonazole group.

\section{Tinea cruris and tinea corporis}

A randomized, multicenter, double-blind, vehicle-controlled Phase III study was conducted to evaluate the efficacy and safety of luliconazole $1 \%$ cream for the treatment of tinea cruris. ${ }^{19}$ A total of 483 participants with $\mathrm{KOH}$-confirmed tinea cruris were randomly allocated in a 2:1 ratio to receive luliconazole cream $1 \%$ or vehicle cream once daily for 7 days.
A total of 256 were evaluated for efficacy. Efficacy assessments were conducted during each visit at days $0,7,14,21$, and 28 of the trial and consisted of the evaluation of clinical signs by investigators on a 4-point scale (0 "no symptoms" to 3 "severe") and skin scrapings for $\mathrm{KOH}$ and culture tests. The primary efficacy endpoint was complete clearance, defined as clinical cure (complete resolution of clinical signs and symptoms) and mycological cure (negative $\mathrm{KOH}$ and culture) at day 28. Secondary efficacy endpoints included clinical cure at day 28 , mycological cure at day 28 , and effective treatment (minimal signs and symptoms plus mycologic cure) at days 7, 14, 21, and 28. The AEs were also monitored.

A significantly higher proportion of participants treated with luliconazole $1 \%$ cream vs vehicle attained complete clearance at day $28(21.2 \%$ vs $4.4 \%, P<0.001)$. A significantly greater number of participants treated with luliconazole $1 \%$ also attained clinical cure $(24.2 \%$ vs $6.6 \%$, $P<0.001)$, mycologic cure $(78.2 \%$ vs $45.1 \%, P<0.001)$, and effective treatment $(43.0 \%$ vs $18.7 \%, P<0.001)$ at day 28 . Two participants in the luliconazole group reported application-site reactions (general application-site reaction, pruritus, and pain) and four participants in the vehicle group reported worsening of tinea cruris. Overall, the safety profile of luliconazole cream $1 \%$ was similar to vehicle.

Luliconazole 1\% cream was compared to sertaconazole $2 \%$ cream and terbinafine $1 \%$ cream in an open-label pilot study. ${ }^{20} \mathrm{~A}$ total of 83 participants with $\mathrm{KOH}$-confirmed tinea corporis or tinea cruris infections were randomized in a 1:1:1 ratio to sertaconazole $2 \%$ cream twice daily for 4 weeks, luliconazole $1 \%$ cream once daily for 2 weeks, or terbinafine $1 \%$ cream once daily for 2 weeks. Primary efficacy was the rate of participants in each treatment group who had clinical signs and symptoms of tinea infection at the end of treatment and at 2-week posttreatment follow-up. Rates of pruritus, erythema, vesiculation, and desquamation were graded from "none" $=0$ to "severe" =3. Mycologic assessment was based on the $\mathrm{KOH}$ wet mount. Secondary endpoints included change in clinical signs and symptom composite score, and the rate of successful treatment based on a PGA score. Treatment-related AEs were also documented.

A total of 62 participants completed the study. At the end of treatment, a higher proportion of participants in the sertaconazole group had resolution of pruritus, erythema, and desquamation compared to erbinafine and luliconazole. The proportion was only significantly higher for pruritus and only when compared to terbinafine but not luliconazole. At follow-up, $100 \%$ of participants in the sertaconazole and 
luliconazole groups and $95.5 \%$ of the participants in the terbinafine group showed absence of pruritus. All the participants in all groups showed absence of erythema, vesicles, and desquamation at follow-up. Furthermore, all participants in the sertaconazole, terbinafine, and luliconazole groups were $\mathrm{KOH}$ negative at the end of treatment and at 2-week posttreatment follow-up. At the end of treatment, participants in the sertaconazole group had a greater reduction in mean composite score (97.1\%) compared to luliconazole $(92.9 \%)$ and terbinafine $(91.2 \%)$. The composite score was zero for all participants in the sertaconazole and luliconazole groups and 0.05 in the terbinafine group. At end of treatment, $100 \%$ of the participants in the sertaconazole group were considered to have successful treatment compared to $95 \%$ of the luliconazole group and $86.4 \%$ of the terbinafine group. All treatments were well tolerated; yet one participant in the sertaconazole group withdrew from the study due to dermatitis

The efficacy of luliconazole, sertaconazole, eberconazole, terbinafine, and amorolfine in treating superficial fungal infections was investigated in a head to head trial. ${ }^{21}$ A total of 150 participants with tinea pedis, tinea corporis, and tinea cruris were randomized in blocks of 30 to receive luliconazole, sertaconazole, eberconazole, terbinafine, and amorolfine. Tinea infections were confirmed by $\mathrm{KOH}$ microscopy. The participants with tinea cruris/corporis were instructed to apply their respective agents for 1 week, while the participants with tinea pedis were instructed to apply the test agent for 2 weeks. Symptoms were assessed at baseline and at 1- and 4-week follow-up. Efficacy endpoints included the mean change in symptom scores from baseline at 2 and 4 weeks, and the proportion of participants with maximum improvement in signs and symptoms at 4 weeks.

Results showed that at 2 weeks, the participants in the luliconazole, sertaconazole, and amorolfine groups had a significant reduction in signs and symptoms, whereas the participants who received terbinafine or eberconazole did not. By 4 weeks, participants in all but the eberconazole group had a significant reduction in symptoms from baseline. Sertaconazole had the highest rates of participants with maximum improvement in signs and symptoms (93.3\%), followed by luliconazole $(86.6 \%)$, amorolfine $(83.3 \%)$, terbinafine (80\%), and eberconazole (73.3\%); however, statistical comparisons of these rates were not reported. The AEs consisted of localized reactions (redness, burning, irritation, peeling, and itching) and were highest with eberconazole (26.6\%) followed by luliconazole $(20 \%)$, terbinafine $(16.6 \%)$, amo- rolfine (13.3\%), and sertaconazole (6.6\%). This study suffers from some disadvantages in that it is not clearly reported, the randomization scheme and measurement was not clear, mycological findings were not presented, and the results were not stratified by site of infection.

\section{Conclusion}

Luliconazole is a novel broad-spectrum imidazole antifungal. Its antifungal ability has also been shown to surpass its commercial counterparts in vitro. The results from Phase III vehicle-controlled studies showed that luliconazole $1 \%$ was significantly more effective than vehicle in rapidly resolving the signs and symptoms of tinea pedis and tinea cruris, in addition to successfully eradicating the underlying fungal infection. When luliconazole 1\% was evaluated against bifonazole $1 \%$ for tinea pedis, no significant difference in the rate of overall cure, resolution of symptoms, or conversion to negative mycology between agents was found, demonstrating that short-duration treatment for 2 weeks with luliconazole was as effective at curing tinea pedis as 4 weeks' treatment with bifonazole.

Two studies compared luliconazole $1 \%$ cream to sertaconazole cream among other topical agents. ${ }^{20,21}$ Both studies found that participants treated with sertaconazole had higher rates of resolution of clinical signs and symptoms compared to luliconazole; yet the results of these studies are questionable due to defects with study design and unclear study conduct and ambiguous reporting. The comparative effectiveness and safety of luliconazole and sertaconazole remains unclear and well-designed head-to-head trials of newer antifungals are warranted to establish these agents' relative efficacy in treating tinea infections.

Despite limited information on luliconazole's efficacy and its efficacy compared to other antifungal medications, the results summarized demonstrate that luliconazole $1 \%$ cream is effective for the treatment of superficial fungal infections, with comparable AEs to existing antifungals. Luliconazole's short duration of treatment (2 weeks for tinea pedis, 1 week for tinea cruris/corporis $)^{11}$ is an advantage given that patient compliance with longer treatment regimens is a challenge. Luliconazole $1 \%$ cream is currently approved for the once-daily treatment of tinea pedis, tinea cruris, and tinea corporis caused by T. rubrum and E. floccosum. ${ }^{11}$ Furthermore, low minimum inhibitory concentration data suggest that luliconazole may be effective in treating infections caused by Candida. ${ }^{13}$ Luliconazole represents a new addition to the imidazole antifungal arsenal, which may help 
abate mounting fungal resistance due to the commonplace use of topical agents and help prevent disease recurrence due to patient nonadherence.

\section{Disclosure}

The authors report no conflicts of interest in this work.

\section{References}

1. Moriarty B, Hay R, Morris-Jones R. The diagnosis and management of tinea. BMJ. 2012;345:e4380.

2. Havlickova B, Czaika VA, Friedrich M. Epidemiological trends in skin mycoses worldwide. Mycoses. 2008;51 Suppl 4:2-15.

3. Hainer BL. Dermatophyte infections. Am Fam Physician. 2003;67: 101-108.

4. Ilkit M, Durdu M. Tinea pedis: The etiology and global epidemiology of a common fungal infection. Crit Rev Microbiol. 2015;41(3):374-388.

5. Khanna D, Bharti S. Luliconazole for the treatment of fungal infections: an evidence-based review. Core Evid. 2014;9:113-124.

6. Gupta AK, Sauder DN, Shear NH. Antifungal agents: an overview. Part I. J Am Acad Dermatol. 1994;30(5 Pt 1):677-698; quiz 698-700.

7. Borgers M. Mechanism of action of antifungal drugs, with special reference to the imidazole derivatives. Rev Infect Dis. 1980;2:520-534.

8. McClellan KJ, Wiseman LR, Markham A. Terbinafine. An update of its use in superficial mycoses. Drugs. 1999;58(1):179-202.

9. Routt ET, Jim SC, Zeichner JA, Kircik LH. What is new in fungal pharmacotherapeutics? J Drugs Dermatol. 2014;13:391-395; quiz 396.

10. Niwano Y, Kuzuhara N, Kodama H, Yoshida M, Miyazaki T, Yamaguchi $\mathrm{H}$. In vitro and in vivo antidermatophyte activities of NND-502, a novel optically active imidazole antimycotic agent. Antimicrob Agents Chemother. 1998;42:967-970.

11. Valeant Pharmaceuticals Inc. LUZU (luliconazole) Cream, $1 \%$ for topical use [Internet]. 2013. Available from: http://www.accessdata.fda. gov/drugsatfda_docs/label/2013/204153s0001bl.pdf. Accessed June 22, 2015.
12. Koga $\mathrm{H}$, Tsuji $\mathrm{Y}$, Inoue $\mathrm{K}$, Kanai $\mathrm{K}$, Majima T, Kasai $\mathrm{T}$, et al. In vitro antifungal activity of luliconazole against clinical isolates from patients with dermatomycoses. J Infect Chemother. 2006;12:163-165.

13. Koga H, Nanjoh Y, Makimura K, Tsuboi R. In vitro antifungal activities of luliconazole, a new topical imidazole. Med Mycol. 2009;47: 640-647.

14. Koga H, Nanjoh Y, Kaneda H, Yamaguchi H, Tsuboi R. Short-term therapy with luliconazole, a novel topical antifungal imidazole, in guinea pig models of tinea corporis and tinea pedis. Antimicrob Agents Chemother. 2012;56(6):3138-3143.

15. Watanabe S, Takahashi H, Nishikawa T, et al. Dose-finding comparative study of 2 weeks of luliconazole cream treatment for tinea pedis comparison between three groups $(1 \%, 0.5 \%, 0.1 \%)$ by a multi-center randomised double-blind study. Mycoses. 2007;50:35-40.

16. Jarratt M, Jones T, Kempers S, et al. Luliconazole for the treatment of interdigital tinea pedis: A double-blind, vehicle-controlled study. Cutis. 2013;91:203-210

17. Jarratt M, Jones T, Adelglass J, et al. Efficacy and safety of once-daily luliconazole $1 \%$ cream in patients $\geq 12$ years of age with interdigital tinea pedis: a phase 3, randomized, double-blind, vehicle-controlled study. J Drugs Dermatol. 2014;13(7):838-846.

18. Watanabe S, Takahashi H, Nishikawa T, et al. A comparative clinical study between 2 weeks of luliconazole $1 \%$ cream treatment and 4 weeks of bifonazole 1\% cream treatment for tinea pedis. Mycoses. 2006;49(3):236-241.

19. Jones TM, Jarratt MT, Mendez-Moguel I, et al. A randomized, multicenter, double-blind, vehicle-controlled study evaluating the efficacy and safety of luliconazole cream $1 \%$ once daily for 7 days in patients aged $\geq 12$ years with tinea cruris. J Drugs Dermatol. 2014;13: $32-38$.

20. Jerajani H, Janaki C, Kumar S, Phiske M. Comparative assessment of the efficacy and safety of sertaconazole $(2 \%)$ cream versus terbinafine cream (1\%) versus luliconazole (1\%) cream in patients with dermatophytoses: a pilot study. Indian J Dermatol. 2013;58(1):34-38.

21. Tamil Selvan A, Gutha Girisha, Vijaybhaskar, Suthakaran R. Comparative evaluation of newer topical antifungal agents in the treatment of superficial fungal infections (tinea or dermatophytic). Int Res J Pharm. 2013;4:224-228.
Infection and Drug Resistance

\section{Publish your work in this journal}

Infection and Drug Resistance is an international, peer-reviewed openaccess journal that focuses on the optimal treatment of infection (bacterial, fungal and viral) and the development and institution of preventive strategies to minimize the development and spread of resistance. The journal is specifically concerned with the epidemiology of antibiotic

\section{Dovepress}

resistance and the mechanisms of resistance development and diffusion in both hospitals and the community. The manuscript management system is completely online and includes a very quick and fair peerreview system, which is all easy to use. Visit http://www.dovepress.com/ testimonials.php to read real quotes from published authors. 